\title{
DNA Cross-Links, Causative Agents and Repairing Agents
}

Taha Nazir ${ }^{1}$, Saeed Ur Rashid Nazir ${ }^{2}$, , Furqan K. Hashmi ${ }^{4}$, Rahat Shamim ${ }^{4}$, Humayun Riaz', Safia Sultana

Munir $^{6}$, Azharul Islam ${ }^{3}$

${ }^{1}$ Microbiology and Molecular Biology Research Group, Advanced Multiple Incorporation, Mississauga ON Canada.

${ }^{2}$ Faculty of Pharmacy, College of Pharmacy, University of Sargodha, Sargodha 40100, Pakistan.

${ }^{3}$ Department of Internal Medicine, University of Texas Medical Branch, Galveston, Texas 77555, USA.

${ }^{4}$ University College of Pharmacy, University of the Punjab, Lahore, Pakistan.

${ }^{5}$ Rashid Latif College of Pharmacy, Lahore, Pakistan.

${ }^{6}$ Professor, Shalamar Medical and Dental College, Lahore, Pakistan.

Authors' Contributions

1 Conception \& Study design, Drafting of

Manuscript, Critical Review.

2 Data Collection \& Processing, Drafting of

Manuscript, Critical Review.

3 and 4 Data Analysis and/or interpretation,

Drafting of Manuscript, Critical Review.

5 Conception \& Study design, Critical Review.

7 Conception \& Study design, Drafting of Manuscript.

\section{Acknowledgement}

\section{Article info}

Received: July 1, 2020

Accepted: January 13, 2021

Funding Source: Nil

Conflict of Interest: Nil

Cite this article: Nazir T, Nazir SUR, Hashmi FK, Shamim R, Riaz H, Munir SS, Islam A.

DNA Cross-Links, Causative Agents and Repairing Agents. RADS J Pharm Pharm Sci. 2020; 8(3):181-185.

*Address of Correspondence Author: srnazir@yahoo.com

\section{ABSTRACT}

Context: deoxyribonucleic acid is under attack continuously by numerous exogenous and endogenous factors. DNA lesions are introduced by the DNA replication. This takes place because of mismatched ribonucleotides or bases that areincorporated randomly by the deoxyribonucleic acid polymerases.

Objectives: The study intended to examine the data from studies onDNA cross-links, causative agents and repairing agents.

Design: The research team performed a literature review, searching relevant literature databases. The sources of data included bioRxiv, medRxiv, Google Scholar, Embase, PsychINFO, and PubMed. The search terms were DNA cross-links, causative and repairing agent.

Setting: The study took place in the main library of the University of Sargodha.

Results: The study identified 39 unique studies that had reported and confirmed DNA cross-links, causativeand repairing agent. The studies found thatthe chemical reliability of Deoxyribonucleic acid is persistently being confronted by environmental and cellular genotoxic agents that create a varied arrangement of covalent nucleobase adduct.

Conclusion: The agents that produce these DRCs include ionizing radiation and UV light. Crosslinking agents are a diverse group of chemical molecules that comprise of two or additional reactive ends. It hasproved that homologous repair and nucleoside excision repair have cooperated closely and mitigated the genotoxic effect of DNA protein cross-links in the cells of Escherichia coli. DNA cross-linking therapies are used to treat various genetic diseases. These lesions can be detected by various methods and repaired using cross-linking agents such ascisplatin, and mitomycin.

Keywords: Protein, Cross-link, Agents and Cisplatin. 


\section{INTRODUCTION}

Human deoxyribonucleic acid is under attack continuously by numerous exogenous and endogenous factors that are estimated to be at the rate of approximately 200,000 actions per cell per day. DNA lesions are also introduced by the DNA replication. This takes place because of mismatched ribonucleotides or bases that are incorporated randomly by the deoxyribonucleic acid polymerases. Furthermore, this process becomes more prone to errors when the deoxyribonucleic acid polymerases repeat more replications [1]. These errors can lead to expansion or shortening of the repeats that gives rise to diseases such as Amyotrophic lateral sclerosis and Huntington's disease. Another source that leads to damage of DNA is endogenous in nature which primes to the natural decay of deoxyribo nucleic acid, such as Deprimidination/depurination of DNA is a spontaneous process that leads to deamination of cytosine and it takes place at an estimated rate of 10,000 and 100-500/cell/day respectively. Moreover, oxidation and methylation of DNA by the cellular reactions byproducts causes roughly around 40,000 and 400-1500 lesions/cell/day. If these lesions are not repaired on time, these can be hazardous to the human health as they can cause several mutations that cause death of cells, cancer and give rise to various genetic disorders [2].

The chemical reliability of Deoxyribonucleic acid is persistently being confronted by environmental and cellular genotoxic agents that create a varied arrangement of covalent nucleobase adduct. If these are left without repairing, these lesions of DNA damage significant cellular procedures, that include regulation of the cell cycle transcription, replication which ultimately leads to mutations, chromosomal rearrangements, and in stability of the genome that intimidates the living of the particular organism and primes to human ailments [3]. The current research team wanted to make available the information onDNA cross -links, causative agents and repairing agents.

\section{METHODS}

\section{Procedures}

The study took place in the main library of the University of Sargodha in Sargodha, Pakistan. The research team searched the relevant literature databases in March 2020. The sources of data included bioRxiv, medRxiv, Google Scholar, Embase, PsychINFO, WanFang Data, and PubMed. These online databases contain archives of the most English and Chinese biomedical journals. The search terms included DNA cross -links, causative agents and repairing agents. Only papers that were published in English were considered, and all available years in each database were searched. The initial search identified 64 published articles, for which the research team obtained full texts that they independently read in full to identify those papers suitable for inclusion in the review. Studies that included the abovementioned terms were all considered. Eventually, 39 unique academic publications were included in the study. Nonscientific commentary, reports, and news articles were excluded from the analysis. Disagreements on the inclusion or exclusion of literature were resolved through discussion. Using a Microsoft Excel spreadsheet, data were extracted from the chosen studies. No blinding regarding a journal or author was done.

\section{DISCUSSION}

\section{DNA-PROTEIN CROSS-LINKS GENERATED BY CHEMICAL AND PHYSICAL AGENTS}

The intact double stranded DNA produces DRCs (DNA protein cross linking) that are induced by exogenous and endogenous agents. The agents that produce these DRCs include ionizing radiation and UV light, free radicals that are generated by cellular metabolism, chemotherapeutic agents like aldehydes and cisplatin, metal complexes that include arsenic, nickel and chromium. Though the details of structural information are not available, the structure details can be concluded from the researches that involve in vitro cross linking of amino acids and the particular nucleotides [4]. An enzymatic system that is efficient relatively with the specificity of broad substrate is human nucleoside excision repair that has no detectable lesions or damage and that does not perturb the structure of DNA to the helix distorting bulky lesions that are induced by chemical carcinogens and $U \mathrm{~V}$ radiation. In the double helix DNA The above mentioned pathway is responsible for the removal and recognition of DCPs [5]. In a research study where in vivo studies were performed using XP-A incision repair deficient cell line suggested that cross links were removed by using 
NER that were induced by platinum but this procedure failed when it was applied for crosslinks that were induced by formaldehyde [6]. Consequently, in another study it was proved that histones cross links $\mathrm{H} 1$ that were mediated by platinum were not in vitro excised by mammalian excision repair system. It can be concluded from these studies cross links induced chemically cannot be considered to be removed by nucleotide excision repair pathway [7].

\section{CROSS LINKING AGENTS}

Crosslinking agents are a diverse group of chemical molecules that comprise two or additional reactive ends. In cells, DNA functional groups can be covalently coupled with molecules by using crosslinking agents that are bi functional in nature [8]. Reactions with DNA may encompass one or both Intra-strand Crosslink helixes to form altered kinds of lesions, containing DNA-protein crosslinks, DNA mono-adducts, interstrand crosslinks and intra-strand crosslinks (ICLs)as shown in Figure 1 and 2 [9]. Though a range of DNA lesions can be created by crosslinking agents in cells, cytotoxicity is habitually attributed to the development of Intra and inter Cross Links. By covalently connecting complementary strands of the double helix of DNA, Inter and intra Cross Links block separation of strand required for essential cellular progressions like DNA transcription and replication [10]. Failure to eliminate Intra and inter Cross Links from DNA can wedge cell cycle development and lead to death of cell. Deficiencies in Inter and intra Cross Link repair can also prime to disastrous chromosomal abnormalities that stimulate tumorigenesis [11].

\section{DNA-Protein Crosslink}

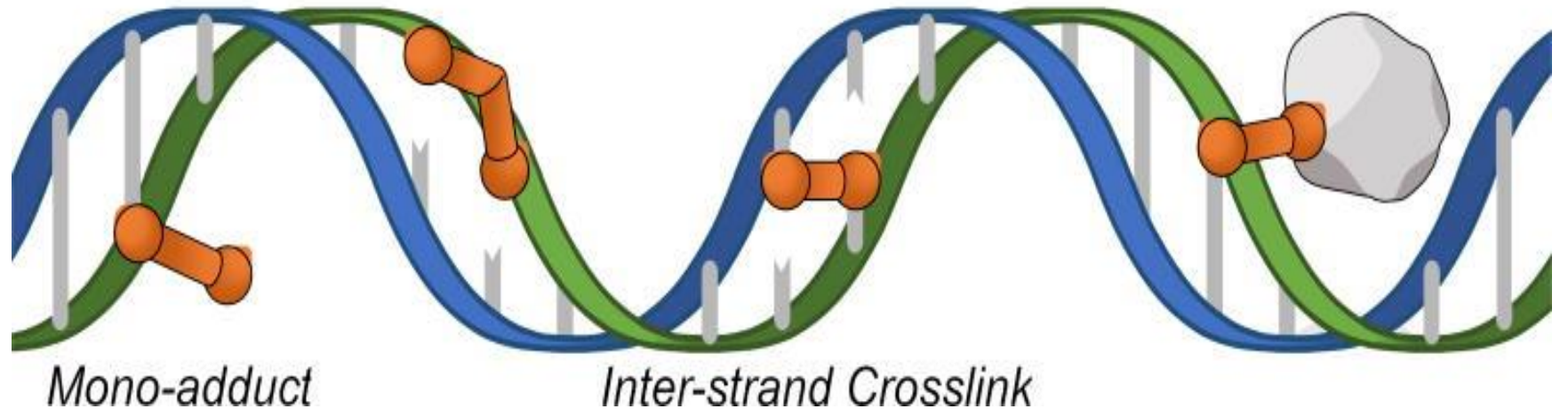

Figure 1. DNA lesions formed by cross-linking agents.

Cross linking agents are divided into four main subgroups that are developed to be used clinically, these are

- Mitomycins (isolated from Streptomyces caespitosis)

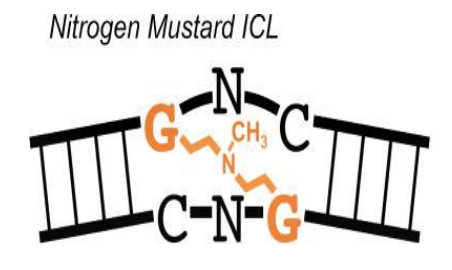

Mitomycin C ICL

- Platinum based compounds (cisplatin, oxaliplatin, carboplatin, satraplatin and nedaplatin)

- Nitrogen mustards (melphalan and cyclophosphamide)

- And psoralens (methoxypsoralen and trimethylpsoralen)

Structural differences show how these agents interact with the DNA and what kind of lesions they create [12].



Psoralen ICL

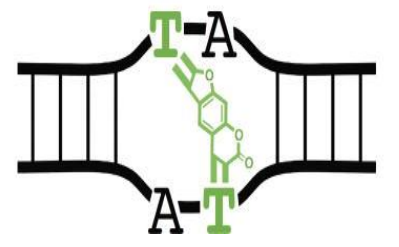

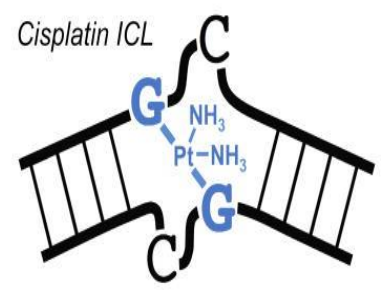

Figure 2. Diversity of ICL Lesions.

\section{BASE EXCISION REPAIR}

Base excision repair is used to remove small adducts typically in which a specific lesion of DNA is hydrolyzed (the $\mathrm{N}$ glyosidic bond) by DNA glycolase the liberates the aberrant nucleobase from the 
phosphoribose backbone [13]. The consequential a basic position is filched at the $5^{\prime}$ side by an apyrimidinic/apurinic (AP) endonuclease to generate a free 3'-hydroxyl group essential for synthesizing of different Deoxyribonucleic acid. In disparity, large and helix misrepresenting lesions are detached by nucleotide excision repair, that includes elimination of an oligonucleotide section comprising the lesion via the action of double nuclease incisions, tracked by helicase elimination of the impaired section [14].

\section{Repairing of DNA Protein Crosslinks in E. coli}

It has been proved by research in vitro and in vivo that homologous repair and nucleoside excision repair have cooperated closely and mitigated the genotoxic effect of DNA protein cross links in the cells of Escherichia coli. The processing of oversized DNA protein cross links is exclusively done by RecBCDdependenthomologous repair whereas the DNA protein crosslinks that are sized between 12-14 kDA are removed with nucleoside excision repair. The size limit of DNA proteins crosslinks that are processed by nucleoside excision repair is dependent upon the UvrB loading efficiency and the recognition of the damaged protein in the nucleoside excision repair on to the DNA protein crosslinks [15]. Depending upon this procedure the nucleoside excision repair incision efficiencyfor the deoxyribonucleic acid that contains the DNA proteins crosslinks diverges considerably with the in vitro peptides and cross-linked proteins size. Fascinatingly no breakage of chromosomes was observed in cells that followed the FA treatment, though the homologous repair of the DNA protein crosslinks had been processed via RecBD pathway, which is specifically for the recombination that have been initiated at the double stands breaks of DNA, so it was proved from this study that E.coli cells had employed both damage and repair tolerance mechanisms for the DNA protein crosslinks. However, numerous damage and repair genes are still to be examined for attaining a clear picture of the tolerance and repair process of the DNA protein crosslinks [16].

DNA cross linking therapies are used to treat various genetic diseases such as in cancer chemotherapy, variousmylomas and genetic diseases [17, 18].

\section{CONCLUSION}

Various physical and chemical agents can create variety of lesions in the structure of DNA, these lesions include mono duct DNA, intra cross links, inter cross links and DNA and protein cross links these lesion can give rise to numerous genetic diseases and other fatal disorders such as cancers, these lesions can be detected by various methods and repaired using cross linking agents such as nitrogen mustard, cisplatin, mitomycin and psoralen inter linking agents, crosslinking agents are also used for treating myelomas and used in chemotherapy. Advance studies are required for exact structural defaults.

\section{REFERENCES}

1. Semlow DR, Zhang J, Budzowska M, Drohat AC, Walter JC. Replication-dependent unhooking of DNA interstrand cross-links by the NEIL3 glycosylase. Cell. 2016;167(2):498-511. e14.

2. Xue M, Kim CS, Healy AR, Wernke KM, Wang Z, Frischling $\mathrm{MC}$, et al. Structure elucidation of colibactin and its DNA cross-links. Science. 2019;365(6457):eaax2685.

3. Jackson SP, Bartek J. The DNA-damage response in human biology and disease. Nature. 2009;461(7267):1071-8.

4. Tomasz M. Mitomycin C: small, fast and deadly (but very selective). Chemistry \& biology. 1995;2(9):575-9.

5. Martens-de Kemp SR, Brink A, van der Meulen IH, De Menezes RX, Te Beest DE, Leemans CR, et al. The FA/BRCA pathway identified as the major predictor of cisplatin response in head and neck cancer by functional genomics. Molecular cancer therapeutics. 2017;16(3):540-50.

6. Fullbright $\mathrm{G}$, Rycenga HB, Gruber JD, Long DT. p97 promotes a conserved mechanism of helicase unloading during DNA cross-link repair. Molecular and cellular biology. 2016;36(23):2983-94.

7. Reardon JT, Cheng Y, Sancar A. Repair of DNAprotein cross-links in mammalian cells. Cell Cycle. 2006;5(13):1366-70.

8. Brulikova L, Hlavac J, Hradil P. DNA interstrand cross-linking agents and their chemotherapeutic potential. Current medicinal chemistry. 2012;19(3):364-85.

9. Rycenga HB, Long DT. The evolving role of DNA inter-strand crosslinks in chemotherapy. Current opinion in pharmacology. 2018;41:20-6.

10. Sawyer SL, Tian L, Kähkönen $M$, Schwartzentruber J, Kircher M, Majewski J, et al. Biallelic mutations in BRCA1 cause a new Fanconi 
anemia subtype. Cancer discovery. 2015;5(2):13542.

11. D'Andrea AD. BRCA1: a missing link in the Fanconi anemia/BRCA pathway. Cancer discovery. 2013;3(4):376-8.

12. Lopez-Martinez D, Liang C-C, Cohn MA. Cellular response to DNA interstrand crosslinks: the Fanconi anemia pathway. Cellular and molecular life sciences. 2016;73(16):3097-114.

13. Krokan HE, Bjørås M. Base excision repair. Cold Spring Harbor perspectives in biology. 2013;5(4):a012583.

14. Mullins EA, Warren GM, Bradley NP, Eichman BF. Structure of a DNA glycosylase that unhooks interstrand cross-links. Proceedings of the National Academy of Sciences. 2017;114(17):4400-5.

15. Gamboa Varela J, Gates KS. Simple, High-Yield Syntheses of DNA Duplexes Containing Interstrand DNA-DNA Cross-Links Between an N
4-Aminocytidine Residue and an Abasic Site. Current Protocols in Nucleic Acid Chemistry. 2016;65(1):5.16. 1-5.. 5.

16. Salem AM, Nakano T, Takuwa M, Matoba N, Tsuboi $\mathrm{T}$, Terato $\mathrm{H}$, et al. Genetic analysis of repair and damage tolerance mechanisms for DNA-protein cross-links in Escherichia coli. Journal of bacteriology. 2009;191(18):5657-68.

17. P Basourakos S, Li L, M Aparicio A, G Corn P, Kim J, C Thompson T. Combination platinum-based and DNA damage response-targeting cancer therapy: evolution and future directions. Current medicinal chemistry. 2017;24(15):1586-606.

18. Bhattacharjee $S$, Nandi $S$. Rare genetic diseases with defects in DNA repair: opportunities and challenges in orphan drug development for targeted cancer therapy. Cancers. 2018;10(9):298.

This is an Open Access article distributed under the terms of the Creative Commons Attribution License (http://creativecommons.org/licenses/by/4.0), which permits unrestricted use, distribution, and reproduction in any medium, provided the original work is properly cited. 